\title{
Consistent Conjectural Variations Equilibrium In A Bilevel Human Migration Model
}

\author{
Vyacheslav V. Kalashnikov, Instituto Tecnológico y de Estudios Superiores (ITESM), Mexico; and Central Economics and \\ Mathematics Institute (CEMI), Moscow, Russia; and Sumy State University, Sumy, Ukraine \\ Nataliya I. Kalashnykova, Universidad Autónoma de Nuevo León (UANL), Mexico; and Sumy State University, Sumy, Ukraine \\ M. Aracelia Alcorta García, Universidad Autónoma de Nuevo León (UANL), Mexico \\ Yazmín G. Acosta Sánchez, Universidad Autónoma de Nuevo León (UANL), Mexico \\ Vitaly V. Kalashnikov, University of Nuevo León (UANL), Mexico,
}

\begin{abstract}
In this paper, we develop a bi-level human migration model using the concept of conjectural variations equilibrium (CVE). In contrast to previous works, here we construct a bi-level programming model. The upper level agents are municipalities of competing locations, whose strategies are investments into infrastructures of the locations (cities, towns, etc.). These investments aim at making the locations more attractive for both the residents and potential migrants from other locations. At the lower level of the model, the present residents (grouped into professional communities) are also considered as potential migrants to other locations. They make their decision where to migrate (if at all) comparing the expected values of the utility functions of the destinations and original locations, which are estimated by taking into account their group's conjectures concerning equilibrium migration flows between the involved locations. Applying a special technique to verify the consistency of the conjectures (influence coefficients), the existence and uniqueness results for the consistent conjectural variations equilibrium (CCVE) are established.
\end{abstract}

Keywords: Bilevel Human Migration Model; Variational Inequality Formulation; Consistency Criterion; Consistent Conjectural Variations Equilibrium

\section{INTRODUCTION}

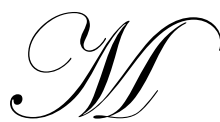

igration problems have been studied actively in many countries throughout the world, as the information / migration prediction / data is extremely useful on a large economic scale. Migration prediction data can stipulate development of facilities necessary to advance employment, education, ecology, etc. Reciprocally, locations with more advanced infrastructure, higher employment capacities, ecology-friendly environment, etc., can attract more potential migrants. On the other hand, overloaded housing/infrastructure facilities may reduce the comfort in the everyday life. Therefore, one can expect a tradeoff in the investments into the locations' infrastructures balanced at a conjectural variations equilibrium state. In the due time period, various migration theories have been developed. In the short historical aspect, one may rely on the excellent fundamental survey Akkoyunlu and Vickerman (2001).

Recently, in the works by Bulavsky and Kalashnikov (1994, 1995), a new array of conjectural variations equilibria (CVE) was introduced and investigated, in which the influence coefficients of each agent affected the structure of the Nash equilibrium. In particular, constant conjectural influence factors were used in the human migration model examined in Isac et al. (2002). More precisely, the potential migration groups were taking into account not only the current difference between the utility function values at the destination and original locations, but also the possible variations in the utility values implied by the change of population volume due to the migration flow. These conjectured variations could be described with an aid of the so-called influence coefficient $w_{i}$. In other 
words, we considered not a perfect competition (with $w_{i}=0$ ) but a generalized Cournot-type model with influence coefficients $w_{i}$ in general different from 1 (in contrast to the classical Cournot model, where $w_{i}=1$ ).

In their previous papers Kalashnikov et al. $(2007,2008)$, the authors extended the latter model to the case where the conjectural variations coefficients may be not only constants, but also functions of the total population at the destination and of the group's fraction in it. Moreover, we allow these functions to take distinct values at the abandoned location and at the destination, which should elevate the model's flexibility. As an experimental verification of the proposed model, we developed a specific form of the model based upon relevant population data of a three-city agglomeration at the boundary of two Mexican states: Durango (Dgo.) and Coahuila (Coah.) Namely, we consider the 1980-2005 dynamics of population growth in the three cities: Torreón (Coah.), Gómez Palacio (Dgo.) and Lerdo (Dgo.), and propose utility functions of three various kinds for each of the three cities. To our knowledge, utility functions of these types were not used in the previous literature dealing with the human migration model. After having collected necessary information about the average movement and transportation (i.e., migration) costs for each pair of the cities, we apply the above-mentioned human migration model to this example. Numerical experiments have been conducted with interesting results concerning the probable equilibrium states revealed.

The main novelty of the recent paper Kalashnykova et al. (2011) lies in the proposed definitions of consistent conjectures and in the outskirt of possible ways to calculate the consistent conjectures and the related consistent conjectural variations equilibrium state (CCVES).

Motivated by the ideas of bi-level structures of processes of migration (the upper level competition among municipalities, and the lower level equilibrium among the potential migrants), we propose a new (bi-level) formulation of the human migration model. Under general enough assumptions, we prove the existence of solutions to the bi-level program as well. Results of numerical experiments (which are underway yet) will be outlined only scarcely.

The paper is organized as follows. Sections 2 through 3, following mainly the previous papers Kalashnikov et al. (2007, 2008) and Kalashnykova et al. (2011), describe the proposed bi-level human migration model, define the conjectural variations equilibrium at the lower level, and cite Theorems 3.1 and 3.2 from Kalashnikov et al. (2007, 2008), which establish the existence and uniqueness of the lower level equilibrium as a solution of an appropriate variational inequality problem. Consistency of conjectures and the existence of the corresponding bilevel equilibrium are discussed in Section 4. Conclusion (Section 5), acknowledgments, and the list of references complete the paper.

\section{PROBLEM STATEMENT AND PRELIMINARIES}

Similar to Isac et al. (2002) and Kalashnikov et al. (2007, 2008), consider a closed economy with:

$n$ locations, denoted by $i$

$K$ classes of population, denoted by $k$

$\bar{Q}_{i}^{k}$ initial fixed population of class $k$ in location $i$

$Q_{i}^{k}$ final population of class $k$ in location $i$

$s_{i j}^{k}$ migration flow of class $k$ from origin $i$ to destination $j$

$c_{i j}^{k}\left(s_{i j}^{k}\right)=b_{i j}^{k} s_{i j}^{k}+\frac{1}{2} a_{i k}^{k}\left(s_{i j}^{k}\right)^{2}$ cost of migration for group $k$ from location $i$ to location $j$

Assume that the migration cost reflects not only the cost of physical movement but also the personal and psychological cost as perceived by a class when moving between locations. The utility $u_{i}^{k}$ (attractiveness of location $i$ as perceived by class $k$ ), depends on the population at destination, that is, $u_{i}^{k}=u_{i}^{k}\left(Q_{i}^{k}\right)$. This assumption is quite 
natural: indeed, in many cases, the cities with higher population provide much more possibilities to find a job, better medical service and household facilities, a developed infrastructure, etc. On the other hand, when the infrastructure development lags behind the modern city demands, the higher population may lead to certain decrease in the living standards, and hence, of the utility values.

These utility functions also incorporate parameters reflecting the scale of investments made by the location's authorities in order to improve the infrastructure, employment capacities, household construction, power supply, and so on. Exactly these amounts of investment play the role of the municipality strategies in the game at the upper level.

First, we describe the lower level problem. The conservation of flow equations, given for each class $k$ and each location $i$, and the inequalities forbidding repeated or chain migration, are listed below:

$$
Q_{i}^{k}=\bar{Q}_{i}^{k}+\sum_{j \neq i} s_{j i}^{k}-\sum_{j \neq i} s_{i j}^{k}, \quad i=1, \ldots, n
$$

and

$$
\sum_{j \neq i} s_{i j}^{k} \leq \bar{Q}_{i}^{k}, \quad i=1, \ldots, n,
$$

with $s_{i j}^{k} \geq 0, \forall k=1, \ldots, K ; j \neq i$. Denote the problem's feasible set by

$$
M=\{(Q, s) \mid s \geq 0,(Q, s) \text { satisfies (1) - (2) }\} .
$$

Equation (1) states that the population of class $k$ at location $i$ is determined by the initial population of class $k$ at location $i$ plus the migration flow into $i$ of that class minus the migration flow out of $i$ for that class. Equation (2) affirms that the flow out of $i$ by class $k$ cannot exceed the initial population of class $k$ at $i$, since no chain migration is allowed.

Assume that migrants are rational, and that migration continues until no individual has any incentive to move, since a unilateral decision will no longer yield a positive net gain (the gain in the expected utility minus the migration cost).

In order to extend the human migration model from Isac et al. (2002), here we introduce the following concepts.

Let $w_{i j}^{k+} \geq 0$ be an influence coefficient taken in account by an individual of class $k$ moving from $i$ to $j$. This coefficient is defined by her assumption that after the movement of $s_{i j}^{k}$ individuals of class $k$ from $i$ to $j$ the total population of class $k$ at $j$ will become equal to:

$\bar{Q}_{j}^{k}+w_{i j}^{k+} s_{i j}^{k}$.

On the other hand, let $w_{i j}^{k-} \geq 0$ be an influence coefficient conjectured by an individual of class $k$ moving from $i$ to $j$, determined by the assumption that after the movement of $s_{i j}^{k}$ individuals, the total population of class $k$ in $i$ will remain equal to:

$\bar{Q}_{i}^{k}-w_{i j}^{k-} s_{i j}^{k}$ 
We accept the following assumptions concerning the utility functions and expected variations of the utility values:

A1. The utility $u_{i}^{k}=u_{i}^{k}\left(Q_{i}^{k}\right)$ is a monotone decreasing and continuously differentiable function.

A2. Each person of class $k$, when considering her possibility of moving from location $i$ to location $j$, takes into account not only the difference in the utility values at the initial location and the destination, but also both the expected (negative) increment of the utility function value at $j$

$$
s_{i j}^{k} w_{i j}^{k+} \frac{\partial u_{j}^{k}}{\partial Q_{j}^{k}}
$$

and the expected (positive) utility value increment in location $i$

$$
-s_{i j}^{k} w_{i j}^{k-} \frac{\partial u_{i}^{k}}{\partial Q_{i}^{k}}
$$

\section{DEFINITION OF EQUILIBRIUM}

\subsection{Definition of Equilibrium in the Lower Level}

A multi-class population and flow pattern $\left(Q^{*}, s^{*}\right) \in M$ is an equilibrium in the lower level, if for each class $k=1, \ldots, K$, and for each pair of locations $i, j=1, \ldots, n ; i \neq j$, the following relationship holds:

$$
u_{i}^{k}-s_{i j}^{k^{*}} w_{i j}^{k-} \frac{\partial u_{i}^{k}}{\partial Q_{i}^{k}}\left(Q^{*}\right)+b_{i j}^{k}+a_{i j}^{k} s_{i j}^{k^{*}}\left\{\begin{array}{l}
=u_{j}^{k}+s_{i j}^{k^{*}} w_{i j}^{k+} \frac{\partial u_{j}^{k}}{\partial Q_{j}^{k}}\left(Q^{*}\right)-\lambda_{i}^{k}, \text { if } s_{i j}^{k^{*}}>0 ; \\
\geq u_{j}^{k}+s_{i j}^{k^{*}} w_{i j}^{k+} \frac{\partial u_{j}^{k}}{\partial Q_{j}^{k}}\left(Q^{*}\right)-\lambda_{i}^{k}, \text { if } s_{i j}^{k^{*}}=0 ;
\end{array}\right.
$$

and

$$
\lambda_{i}^{k} \begin{cases}\geq 0, & \text { if } \quad \sum_{l \neq i} s_{i l}^{k^{*}}=\bar{Q}_{i}^{k} ; \\ =0, & \text { if } \sum_{l \neq i} s_{i l}^{k^{*}}<\bar{Q}_{i}^{k} .\end{cases}
$$

A3. We assume that the influence coefficients are functions depending upon the current population at the location in question and the migration flow from location $i$ to location $j$, satisfying the following conditions:

$$
s_{i j}^{k} w_{i j}^{k+}(Q, s)=v_{i j}^{k+} s_{i j}^{k}+\sigma_{i j}^{k+} Q_{j}^{k},
$$

and

$$
s_{i j}^{k} w_{i j}^{k-}(Q, s)=v_{i j}^{k-} s_{i j}^{k}-\sigma_{i j}^{k-} Q_{i}^{k},
$$

where

$$
v_{i j}^{k \pm} \geq 0, \quad \sigma_{i j}^{k \pm} \geq 0, \quad k=1, \ldots, J ; \quad i \neq j .
$$


Taking into account assumption A3 and omitting for shortness the argument $Q *$ in the utility functions, we turn (3) into:

$$
u_{i}^{k}-s_{i j}^{k^{*}} v_{i j}^{k-} \frac{\partial u_{i}^{k}}{\partial Q_{i}^{k}}+\sigma_{i j}^{k-} Q_{i}^{k^{*}} \frac{\partial u_{i}^{k}}{\partial Q_{i}^{k}}+b_{i j}^{k}+a_{i j}^{k} s_{i j}^{k^{*}}=u_{j}^{k}+s_{i j}^{k^{*}} v_{i j}^{k+} \frac{\partial u_{j}^{k}}{\partial Q_{j}^{k}}+\sigma_{i j}^{k+} Q_{j}^{k^{*}} \frac{\partial u_{j}^{k}}{\partial Q_{j}^{k}}-\lambda_{i}^{k}, \text { if } s_{i j}^{k^{*}}>0 ;
$$

and

$$
u_{i}^{k}-s_{i j}^{k^{*}} v_{i j}^{k-} \frac{\partial u_{i}^{k}}{\partial Q_{i}^{k}}+\sigma_{i j}^{k-} Q_{i}^{k^{*}} \frac{\partial u_{i}^{k}}{\partial Q_{i}^{k}}+b_{i j}^{k}+a_{i j}^{k} s_{i j}^{k^{*}} \geq u_{j}^{k}+s_{i j}^{k^{*}} v_{i j}^{k+} \frac{\partial u_{j}^{k}}{\partial Q_{j}^{k}}+\sigma_{i j}^{k+} Q_{j}^{k^{*}} \frac{\partial u_{j}^{k}}{\partial Q_{j}^{k}}-\lambda_{i}^{k}, \text { if } s_{i j}^{k^{*}}=0
$$

Assume that the utility function associated with a particular location and a single class can depend upon the population associated with every class and each location, that is, compose a vector-function $u=u(Q)$. Assume also that the cost associated with migration between two locations as perceived by a particular class can depend, in general, upon the flow of each class between every pair of locations, i.e., compose an aggregate vector-function $c=c(s)$. Finally, let us compose an auxiliary vector of the appropriate size as follows:

$$
d(Q, s)=\left(d_{i j}^{k}(Q, s)\right) \text {, where } d_{i j}^{k}(Q, s)=s_{i j}^{k} v_{i j}^{k-} \frac{\partial u_{i}^{k}}{\partial Q_{i}^{k}}-\sigma_{i j}^{k-} Q_{i}^{k} \frac{\partial u_{i}^{k}}{\partial Q_{i}^{k}}+s_{i j}^{k} v_{i j}^{k+} \frac{\partial u_{j}^{k}}{\partial Q_{j}^{k}}-\sigma_{i j}^{k+} Q_{j}^{k} \frac{\partial u_{j}^{k}}{\partial Q_{j}^{k}}
$$

Now we are in a position to formulate the following result, established in the previous papers Kalashnikov et al. (2007, 2008):

Theorem 3.1. A population and migration flow pattern $\left(Q^{*}, s^{*}\right) \in M$ satisfies the equilibrium conditions (3) and (4) if, and only if it solves the variational inequality problem

$$
\left\langle-u\left(Q^{*}\right), Q-Q^{*}\right\rangle+\left\langle c\left(s^{*}\right)-d\left(Q^{*}, s^{*}\right), s-s^{*}\right\rangle \geq 0, \forall(Q, s) \in M
$$

The existence of at least one solution to the variational inequality (6) follows from the general theory of variational inequalities, under the sole assumption of continuous differentiability of the utility functions $u$ and continuity of migration cost functions $c$, since the feasible convex set $M$ is compact ( $c f$., for example, Kinderlehrer and Stampacchia, 1980).

From now on, we omit the superscript $k$ for simplicity purpose. The uniqueness of the equilibrium population and migration flow pattern $\left(Q^{*}, s^{*}\right)$ follows under the assumption that the compound operator

$$
\left(\begin{array}{c}
-u(Q) \\
c(s)-d(Q, s)
\end{array}\right): R^{K \times n} \times R^{K \times n \times(n-1)} \rightarrow R^{K \times n} \times R^{K \times n \times(n-1)},
$$

involving the utility and migration cost functions, is strictly monotone over the feasible set $M$ :

$$
\left\langle\left(\begin{array}{l}
-u\left(Q^{1}\right) \\
c\left(s^{1}\right)-d\left(Q^{1}, s^{1}\right)
\end{array}\right)-\left(\begin{array}{l}
-u\left(Q^{2}\right) \\
c\left(s^{2}\right)-d\left(Q^{2}, s^{2}\right)
\end{array}\right),\left(\begin{array}{l}
Q^{1}-Q^{2} \\
s^{1}-s^{2}
\end{array}\right)\right\rangle>0, \quad \forall\left(\begin{array}{c}
Q^{1} \\
s^{1}
\end{array}\right) \neq\left(\begin{array}{c}
Q^{2} \\
s^{2}
\end{array}\right),
$$


that is,

$$
-\left\langle u\left(Q^{1}\right)-u\left(Q^{2}\right), Q^{1}-Q^{2}\right\rangle+\left\langle c\left(s^{1}\right)-c\left(s^{2}\right), s^{1}-s^{2}\right\rangle-\left\langle d\left(Q^{1}, s^{1}\right)-d\left(Q^{2}, s^{2}\right), s^{1}-s^{2}\right\rangle>0 .
$$

The latter is a consequence of the classical result of the Theory of Variational Inequality Problems (see, for example, Kinderlehrer and Stampacchia, 1980):

Theorem 3.2. Consider the variational inequality: Find $y^{*} \in M \subset R^{n}$ such that,

$$
\left\langle F\left(y^{*}\right), y-y^{*}\right\rangle \geq 0, \quad \forall y \in M
$$

If the operator $F: R^{n} \rightarrow R^{n}$ is strictly monotone, that is,

$$
\left\langle F\left(y^{1}\right)-F\left(y^{2}\right), y^{1}-y^{2}\right\rangle>0, \quad \forall y^{1} \neq y^{2},
$$

then the variational inequality (8) has at most one solution.

Having established the existence and uniqueness of the (lower level) equilibrium among the potential migrants, we may pass to the concept of the upper level equilibrium among the municipal authorities.

\subsection{Definition of Equilibrium in the Upper Level}

We assume that the utility function associated with location $i$ and class $k$ of potential migrants has the form

$$
u_{i}^{k}\left(Q_{i}\right)=A_{i}^{k}-\frac{B_{i}^{k}}{R_{i}} Q_{i}
$$

where $A_{i}^{k}>0, B_{i}^{k}>0$ are parameters related to the environment facilities for the potential immigrants of class $k$ in location $i$. For instance, the economical sense of $A_{i}^{k}$ could be the average cost of a household in location $i$ in a district where the typical representatives of class $k$ prefer to settle down, while $B_{i}^{k}$ might be interpreted as an inverse adaptability coefficient for the immigrants of class $k$ : that is, the lower value of $B_{i}^{k}$, the higher the degree of adaptability revealed by the average family of the specimen of class $k$ to the growing population of location $i$. Finally, the parameter $R_{i}>0$ reflects the amount of investment by the authorities of location $i$ into the improvement of the environment for the newcomers and the regular inhabitants: the higher the investment's amount, the lower the negative effect of the growing population on the location's attractiveness for both the current and potential inhabitants.

Now assuming that the investment volumes $R_{i}>0$ are used as strategies of the players (municipal authorities of the locations involved), it is standard to define an equilibrium state in the (upper level) game.

An investment vector $R^{*}=\left(R_{1}^{*}, R_{2}^{*}, \ldots, R_{n}^{*}\right)$ is an equilibrium in the upper level, if for each location $i, i=$ $1, \ldots, n$, the municipal authority's utility function $U_{i}=U_{i}\left(R_{i}, R_{-i}^{*}\right)$ attains its maximum value exactly at $R_{i}=R_{i}^{*}$ (assuming that all the other players stick to their investment values $R_{-i}^{*}=\left(R_{1}^{*}, \ldots, R_{i-1}^{*}, R_{i+1}^{*}, \ldots, R_{n}^{*}\right)$ ). 
Here, the municipality utility function $U_{i}=U_{i}(R)$ is the weighted sum of the location's utility functions of all the classes of potential migrants determined below:

$$
U_{i}(R)=\frac{Q_{i}^{1^{*}}}{Q_{i}^{*}} u_{i}^{1}\left(Q^{*}\right)+\ldots+\frac{Q_{i}^{K^{*}}}{Q_{i}^{*}} u_{i}^{K}\left(Q^{*}\right)
$$

where $Q^{*}$ is the equilibrium of the lower level population values, which (due to Theorems 3.1 and 3.2) exist uniquely for any (fixed) vector of investments $R$ involved into the structure of locations' utility functions (9).

\section{EXISTENCE OF A BI-LEVEL EQUILIBRIUM WITH CONSISTENT CONJECTURES}

The consistency of conjectures (or, the influence coefficients) arises naturally as an important issue. Indeed, the existence of at least one equilibrium for arbitrary influence coefficients obliges one to select some justified conjectures so that the above concept of the equilibrium make sense. In this section, we propose a concept of consistency and formulate the existence result for the consistent conjectural variations equilibrium.

Based upon the consistency criterion proposed in Kalashnikov et al. (2011), we formulate the following definition. Here, for simplicity, we repeat our assumption that the utility function for each location $i$ and each potential migrant group $k$ is linear of the form $u_{i}^{k}\left(Q_{i}^{k}\right)=A_{i}^{k}-\frac{B_{i}^{k}}{R_{i}} Q_{i}^{k}$, with $A_{i}^{k}>0, B_{i}^{k}>0, R_{i}>0$; next, $a_{i j}^{k}>0$ for each quadratic migration cost function; and finally, conjectures (influence coefficients) are constant, i.e., $\sigma_{i j}^{k, \pm}=0$, and $v_{i j}^{k+}=v_{i j}^{k-}=v_{i j}^{k} \geq 0$, for all $i, j, k$.

Definition 4.1. At a lower level equilibrium (LLE) pattern $\left(Q^{*}, s^{*}\right) \in M$, the influence coefficients $v_{i j}^{k}, k=1, \ldots, K ; i, j=1, \ldots, n ; i \neq j$, are referred to as consistent, if the following equalities hold:

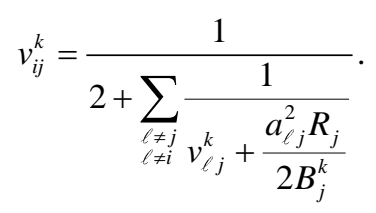

The LLE with consistent conjectures is called a consistent conjectural variations equilibrium state (CCVES) in application to the above-described human migration model.

Now we are in a position to formulate the following existence result.

Theorem 4.1. Under assumptions A1, $A 2$ and A3, and if all the investment sums are bounded (i.e., $\left.0<R_{i} \leq R, \forall i=1, \ldots, n\right)$, then there exists a consistent conjectural equilibrium state (CCVES) in application to the above-described bi-level human migration model.

When proving Theorem 4.1, we established that certain infinite-dimensional mappings involved in equations (11) are continuous and contracting over corresponding compact subsets. This allows one to find, for each fixed group $k$ of potential migrant and each destination location $j$, good approximations for the consistent conjectures (influence coefficients) $v_{i j}^{k}, k=1, \ldots, K ; i, j=1, \ldots, n ; i \neq j$, by applying a simple iteration procedure: 


$$
v_{i j}^{k,(m+1)}=\frac{1}{2+\sum_{\substack{\ell \neq j \\ \ell \neq i}} \frac{1}{v_{\ell j}^{k,(m)}+\frac{a_{\ell j}^{2} R_{j}}{2 B_{j}^{k}}}}, m=0,1, \ldots
$$

with $v_{i j}^{k,(0)}=0, k=1, \ldots, K ; i, j=1, \ldots, n ; i \neq j$.

Theorem 4.2. For each fixed group $k$ of potential migrants and each destination location $j$, approximate conjectures (influence coefficients) $v_{i j}^{k,(m)}, i, j=1, \ldots, n ; i \neq j$, obtained by formulas (12), converge (as $\left.m \rightarrow \infty\right)$ to the unique solution $v_{j}^{k}=\left(v_{i j}^{k}\right)_{i=1, i \neq j}^{n}$ of system (11).

In our future research, we are going to extend the obtained results to the case of not necessarily linear utility functions and discontinuous conjectures (influence coefficients). However, some of the necessary technique can be developed now, in the case of linear utilities and continuous conjectures. To do that, for fixed values of $k$ and $j$, we denote the value of the inverse of the derivative of the utility function $u_{j}^{k}=u_{j}^{k}\left(Q_{j}^{k}\right)$ by

$$
\tau=\left[\frac{d u_{j}^{k}}{d Q_{j}^{k}}\left(Q_{j}^{k}\right)\right]^{-1}<0,
$$

and rewrite the consistency equalities (11) in a more general form:

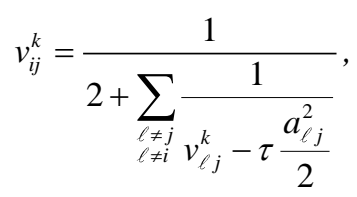

where $\tau \in(-\infty, 0]$. When $\tau \rightarrow-\infty$, then the solution of system (14) tends to the unique limit solution $v_{i j}^{k}=\frac{1}{2}, k=1, \ldots, K ; i, j=1, \ldots, n ; i \neq j$. For all the finite values of the parameter $\tau \leq 0$ we establish the following assertion.

Theorem 4.3. For each fixed group $k$ of potential migrants and each destination location $j$, and for any $\tau \in(-\infty, 0]$, there exists a unique solution of equations (14) as a collection of continuous functions $v_{i j}^{k}=v_{i j}^{k}(\tau), i, j=1, \ldots, n ; i \neq j$. Furthermore, $v_{i j}^{k}(0)=0$, and $v_{i j}^{k}(\tau) \rightarrow \frac{1}{2}$ as $\tau \rightarrow-\infty$, for all $i, j=1, \ldots, n ; i \neq j$.

\section{CONCLUSIONS AND FUTURE RESEARCH}

We have investigated a human migration model involving conjectures of the migration groups concerning the variations of utility function values both in the abandoned location and in the destination site. To formulate equilibrium conditions in this model, we use the concept of conjectural variation equilibrium (CVE). We establish the existence and uniqueness results for the equilibrium in question, and introduced a concept of consistent conjectures (influence coefficients), together with the corresponding CVEs. The theorem guaranteeing the existence and uniqueness of solution to each consistency system and hence, the consistent conjectural variations equilibrium state (CCVES), has been also proved. 
We also notice that the human migration model with conjectural variations can be further extended and examined in the case when constraint (2) is replaced by a weaker condition, say

$$
Q_{i}^{k} \geq 0, i=1, \ldots, n ; k=1, \ldots, K,
$$

which allows us to consider the repeated or chain migration. In this case the feasible set $M$ stops being compact (remaining, however, convex), which makes insufficient the use of the general theory of variational inequality problems to demonstrate the existence of equilibrium. Then subtler results obtained in Bulavsky et al. (1998) and further developed in Isac et al. (2002) can be used to that effect. Indeed, the existence of equilibrium will be guaranteed for various classes of utility functions and migration costs that are free of exceptional families of elements (EFE).

\section{ACKNOWLEDGEMENTS}

The first author's research activities were financially supported by the R\&D Department (Cátedra de Investigación) CAT-174 of the Instituto Tecnológico y de Estudios Superiores de Monterrey (ITESM), Campus Monterrey and by the SEP-CONACYT grant CB-2008-01-106664, Mexico. The second and the fifth authors were also supported by the SEP-CONACyT grant CB-2009-01-127691, and the PAICYT project CE250-09, Mexico.

\section{AUTHOR INFORMATION}

Vyacheslav V. Kalashnikov received a $\mathrm{PhD}$ degree in Operations Research from Institute of Mathematics of the Siberian Division of the USSR Academy of Sciences in Novosibirsk, in 1981. He received the Dr. Sc. (Habilitation) Degree in 1995 from the Central Economics and Mathematics Institute of the Russian Academy of Sciences, Moscow. He is a Professor of Department of Systems and Industrial Engineering at ITESM, Campus Monterrey, Mexico. His present research interests include solution of variational inequality and complementarity problems and applications. He is a member of the National Roster of Researchers in Mexico, American Mathematical Society, Society for Industrial and Applied Mathematics. E-mail: kalash@itesm.mx. Corresponding author.

Nataliya I. Kalashnykova received a PhD degree in Operations Research in 1989 from Institute of Mathematics of the Siberian Division of the USSR Academy of Sciences in Novosibirsk. She has worked as Associate Professor of Department of Mathematical Economics Methods at Sumy State University, Sumy, Ukraine, and at Department of Physics and Mathematics of Autonomous University of Nuevo León (UANL), Monterrey, Mexico. Her present research interests include development of conjectural variations equilibrium (CVE) theory and its applications to electricity markets. She is a member of the National Roster of Researchers (SNI), level I, and Society of Mathematics of Mexico (SMM). E-mail: nkalash@fcfm.uanl.mx

María Aracelia Alcorta García received a B.S. degree in Mathematics in 1987, the M.S degree in Quality Control in 1995 and the Ph.D. degree in Physical Industrial Engineering in 2003, from Universidad Autónoma de Nuevo Leon (UANL), Mexico. She is a member of the National Roster of Researchers (SNI), level I, and now works as professor in the Department of Graduate of the Faculty of Physical and Mathematics Sciences in the UANL. Her research interests focus on polynomial filtering and polynomial stochastic control and optimization. She has been the coordinator of Graduate School of Science with Orientation in Mathematics since 2010. E-mail: aalcorta@fcfm.uanl.mx

Yazmín Guadalupe Acosta Sánchez received a B.S. degree in Mathematics from Universidad Autónoma de Nuevo Leon (UANL), Mexico, in 2005. Her thesis was later published in the International Journal of Innovative Computing, Information and Control with the title "Optimal risk-sensitive approach to filtering and control for linear stochastic systems". Now she continues her education at the Graduate School of Physics and Industrial Engineering of UANL developing models of bilevel equilibrium in human migration models. E-mail: lic_acosta9@ hotmail.com

Vitaliy V. Kalashnikov received a M.S. degree in Mathematics from Moscow State University, Russia, in 2000, the M.S. degree in Financial Mathematics from University of Kaiserslautern, Germany, in 1999, and the Ph.D. degree in 
Economics from University of Alexander Humboldt, Berlin, Germany, in 2009. Now he is a professor at the Graduate School of Economics of the Autonomous University of Nuevo León (UANL), Mexico. His research interests lay primarily in the area of models of oligopoly, conjectural variations equilibrium (CVE), and its application to the study of processes of liberalization in the energy markets (electricity, natural gas, etc). E-mail: kalashnikov_de@yahoo.de

\section{REFERENCES}

1. Akkoyunlu, S., and Vickerman, R. (2001), Migration and the Efficiency of European Labour Markets, Working Paper, Department of Economics, The University of Kent at Canterbury.

2. Bulavsky, V. A., Isac, G., and Kalashnikov, V. V. (1998), Application of topological degree theory to complementarity problems. - In: Multilevel Optimization: Algorithms and Applications, A. Migdalas, P.M. Pardalos and P. Värbrand (Eds.), Kluwer Academic Publishers, Dordrecht/Boston/London, pp. 333-358.

3. Bulavsky, V. A. and Kalashnikov, V. V. (1994), One-parametric driving method to study equilibrium, Economics and Mathematical Methods (Ekonomika i Matematicheskie Metody, in Russian), vol.30, no.2, pp.129-138.

4. Bulavsky, V.A. and Kalashnikov, V.V. (1995), Equilibria in generalized Cournot and Stackelberg models. Economics and Mathematical Methods (Ekonomika i Matematicheskie Metody, in Russian), vol.35, no.3, pp.164-176.

5. Isac, G., Bulavsky, V. A. and Kalashnikov, V. V. (2002), Complementarity, Equilibrium, Efficiency and Economics, Kluwer Academic Publishers, Dordrecht/Boston/London.

6. Kalashnikov, V. V., Kalashnykova, N. I., Luévanos, R., Uranga, C., Méndez, M., and Luévanos, A. (2007), Un modelo de migración humana: Experimentos numéricos basados sobre los datos de las tres ciudades Laguneras, Estudios Demográficos y Urbanos, Colegio de México, vol. 22, no. 3, pp. 731-760.

7. Kalashnikov, V. V., Kalashnykova, N. I., Luévanos, R., Uranga, C., Méndez, M., and Luévanos, A. (2008)

8. Numerical experimentation with a human migration model, European J. Oper. Res., vol. 189, issue 1, pp. 208-229.

9. Kalashnikov, V. V., Bulavsky, V. A., Kalashnykova, N. I., and Castillo, F. J. (2011), Mixed oligopoly with consistent conjectures, European J. Oper. Res., vol. 210, issue 3, pp. 729-735.

10. Kalashnykova, N. I., Kalashnikov, V. V., and Chávez Delgadillo, L. R. (2011), Consistent conjectures in a human migration model: Definition, existence and computation, International Journal of Innovative Computing, Information and Control, ICIC International, Japan, vol. 7, no. 4, pp. 1949-1957.

11. Kinderlehrer, D., and Stampacchia, G. (1980), An Introduction to Variational Inequalities and Their Applications, Academic Press, New York. 